\title{
MANUFACTURE AND ASSEMBLY OF THE 6-METER-LONG CRYOMODULES FOR SUPERCONDUCTING RF TEST FACILITY (STF) AT KEK
}

\author{
Tomoyuki Semba, Soji Kajiura, Takashi Masumoto, Toshiyuki Tagawa, Yutaka Itou \\ Hitachi Ltd., Ibaraki, Japan \\ Hitoshi Hayano, Hiromi Hisamatsu, Mika Masuzawa, Shuichi Noguchi, \\ Norihito Ohuchi, Kenji Saito, Akio Terashima, Kiyosumi Tsuchiya \\ KEK, Ibaraki, Japan
}

\section{Abstract}

A superconducting RF test facility (STF) is being constructed at KEK for R\&D oriented toward the International Linear Collider (ILC). [1] Under KEK's guidance, Hitachi, Ltd. has manufactured cryostat components at its factory and completed final assembly in the STF Linac Building at KEK. The STF cryomodule consists of two cryostats, each about six meters long based on the TESLA-TTF-type III design. Each cryostat can house four 9-cell cavities, each one meter in length. [2] This report outlines the flow of manufacturing from the factory to final assembly, and describes the manufacturing of equipment, assembly precision, and other matters.

\section{EQUIPMENT CONFIGURATION}

This paper first briefly describes the components and specifications of the STF cryomodule.

\section{Overall Configuration}

Figure 1 is an overall diagram of the STF cryomodule; Figure 2 shows the cross-sectional structure. The equipment is characterized by the connection of two relatively short cryostats to efficiently conduct performance tests on two different kinds of cavities. The major components of those cryostats are vacuum vessels, support posts, $80 \mathrm{~K}$ radiation shields, $5 \mathrm{~K}$ radiation shields, a helium gas return pipe, cryogenic piping, cavity helium vessels, RF input couplers, and various types of measuring devices and sensors.

Vacuum vessels, cryogenic piping, support posts, and radiation shields are based on TTF design, whereas cavities, input couplers, tuners, and WPM have been designed by KEK.

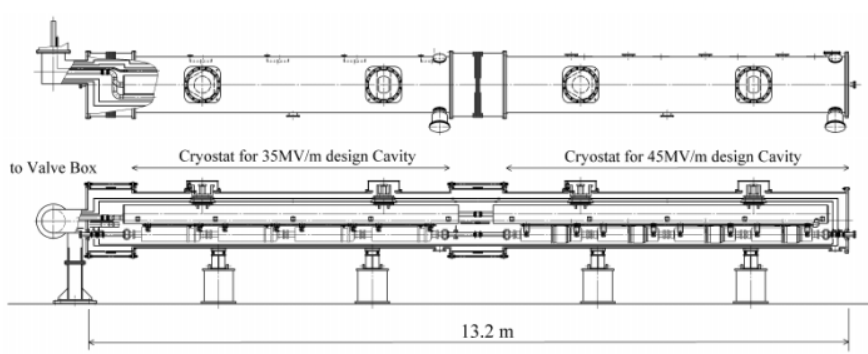

Figure 1: Overview of the STF cryomodule

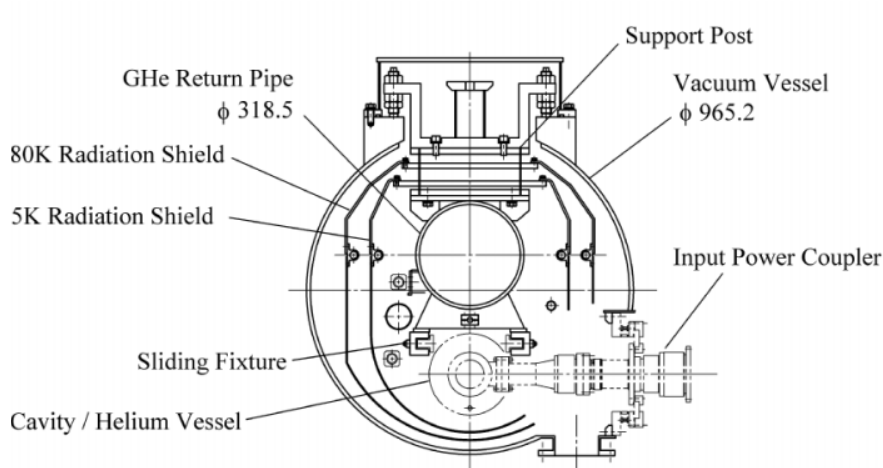

Figure 2: Cross section of the STF cryostat

\section{Equipment Specifications}

The STF cryomodule consists of two cryostats having different detailed specifications designed to test two different kinds of cavities: a baseline $(35 \mathrm{MV} / \mathrm{m})$ cavity an enhanced version of the TTF cavity - and a newly designed LL-type $45 \mathrm{MV} / \mathrm{m}$ cavity. The specifications of these components are described as follows:

- Vacuum vessels: These vacuum vessels have an outer diameter of $965.2 \mathrm{~mm}$. The cryostat for the $35 \mathrm{MV} / \mathrm{m}$ design cavity and the one for the $45 \mathrm{MV} / \mathrm{m}$ design cavity are 5.6 meters and 5.9 meters in length, respectively. Upon final assembly in KEK's underground tunnel, both vacuum vessels are connected via a large-diameter bellows, then overall length becomes 13.2 meters. The vessels are made of carbon steel $12-\mathrm{mm}$ thick to provide protection against geomagnetism.

- Cryogenic piping: Gas helium return pipe (GRP) made of SS316L and $318.5 \mathrm{~mm}$ in diameter is arranged on top of the center part of the cross section, and thus also functions as a support structure for the entire cold mass. The GRP will support the cavity weight (about $100 \mathrm{~kg}$ per cavity) almost as a uniformly distributed load. Figure 3 illustrates a mesh used for related strength analysis. The vertical deflection of the GRP in supporting the load is $0.05 \mathrm{~mm}$ from the resultant load, and therefore is sufficiently small relative to required positional precision in the vertical direction of the beam axis. 


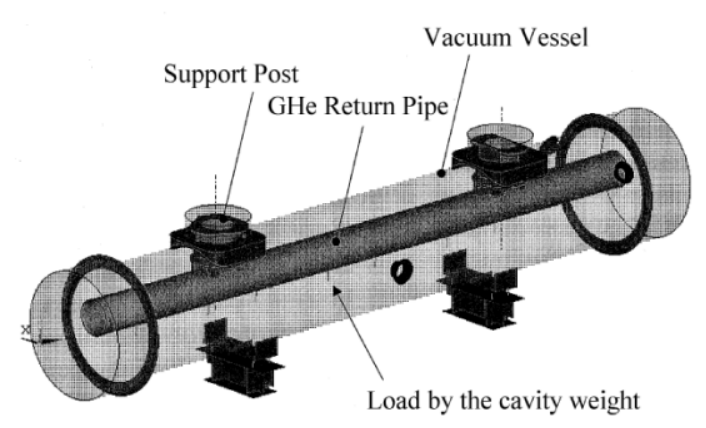

Figure 3: Mesh modeling for structural analysis

The cryogenic piping also includes helium piping made of SS316L and shield cryogenic piping made of aluminum.

- Support posts: These cylindrical heat-insulated supports made of GFRP feature the same specifications as those used in the TTF. INFN-Milano supplied these support posts to KEK, this time. For future purposes, the same type of support post has been prototyped and evaluated at KEK as well.

- Radiation shields: These dual shields are made of aluminum for $5 \mathrm{~K}$ and $80 \mathrm{~K}$, respectively. These shields were designed to connect the cryogenic piping with the shield plates by simply tightening bolts in order to minimize welding work at on-site assembly.

- Cavities, input couplers, tuners, and WPM: These are originally designed by KEK, and manufactured and mounted on the equipment in conjunction with related manufacturers.

\section{MANUFACTURING}

\section{Parts Manufacturing}

Each item of equipment was manufactured on a part-by-part basis at a factory. While the vacuum vessels are large welded structures, the flange faces on which to mount the input couplers had to satisfy a positional precision of $< \pm 2 \mathrm{~mm}$ and an inclination precision of $<$ $2.5 \mathrm{mrad}$. A large, high-precision milling machine was therefore used to machine the flange faces. Since the GRP is the key component as the backbone in the cryomodule, the seats for connecting the support posts and the parts for hanging the cavities were welded onto the GRP. After welding process, the entire pipe with these components was machined to minimize the effects of weld-induced deformations on dimensional precision..

To manufacture the radiation shield plates, aluminum plates were bent and then partially welded. The structure is such that, upon final assembly, the high-rigidity upper half of the thick plate will support the entire shield plate; conversely, the lower half will flexibly follow the overall displacement.

\section{Specific Evaluation Tests}

While each part was manufactured at a factory, important parts were subjected to performance evaluation. The cavity slide mechanism was subjected to a loaded sliding test in $\mathrm{LN}_{2}$, the support posts to tensile and shear strength tests, and dissimilar metal joints to leakage tests with super-fluid helium, among other tests. All these tests verified that the parts have sufficient performance as the cryostats for the STF.

\section{ASSEMBLY}

After being manufactured, the components underwent final assembly at the STF Linac Building at KEK. Figure 4 shows the components being assembled. The vacuum vessels, radiation shield plates, and GRP are shown from the left.

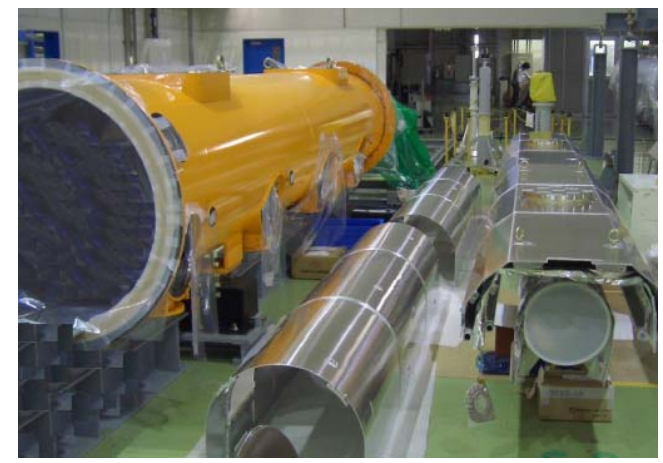

Figure 4: Components of STF cryostat

\section{Cold Mass Assembly}

The cold mass to be inserted into the vacuum vessels was assembled while being suspended under a special-purpose gate-shaped jig. Figure 5 shows this assembly process.

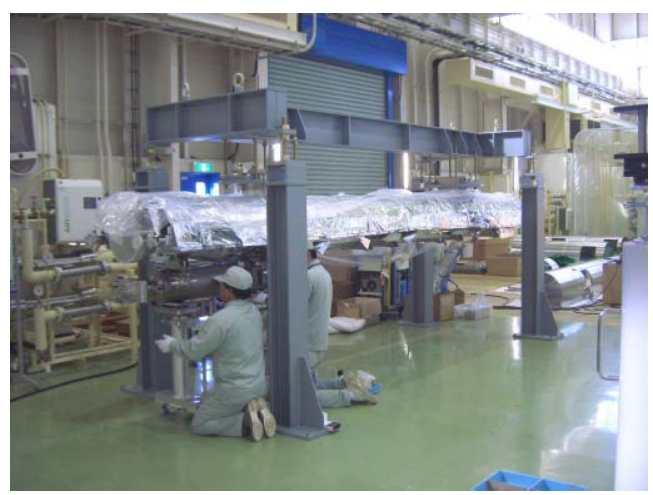

Figure 5: Cold mass assembly

First, the GRP was placed on a gate-shaped jig. At this stage, the GRP was positioned and leveled with a precision of $2 \mathrm{~mm}$ or less in the direction of the beam axis, and $0.1 \mathrm{~mm}$ or less in the vertical direction. Then the cryogenic piping, cavities, measuring devices, and related parts were installed.

The cavities are suspended from the GRP, and this 
region has a cavity slide mechanism in the axial direction. This structure, similar to that of the slide mechanism of the TTF, is such that C-type parts and slide rollers are installed at the tip of the cavity suspension seat, and the pad attached on each cavity helium vessel is sandwiched from three directions to secure equipment positioning in the cross section, while allowing it to slide freely in the direction of the beam axis. Figure 6 shows the cavity slide mechanism.

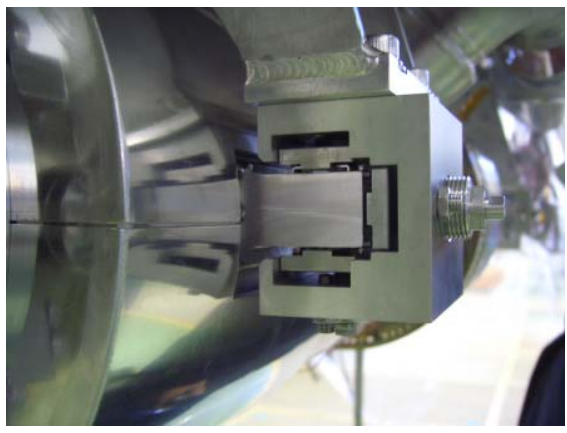

Figure 6: Cavity sliding fixtures

Next in the assembly process are various steps taken in this order: helium leakage test, installation of measuring devices, electrical tests, and other steps. In parallel, KEK will install the WPM detectors and aluminum shield piping for the wires. Finally, the bottom shield plate will be installed to complete the cold mass.

\section{Insertion into the Vacuum Vessels}

The assembled cold mass will be suspended on an assembly jig and then carefully inserted into the vacuum vessels by using another special-purpose jig. After the insertion, metal fixers will be installed on top of the support posts to complete one cryostat. Figure 7 illustrates how this step is taken.

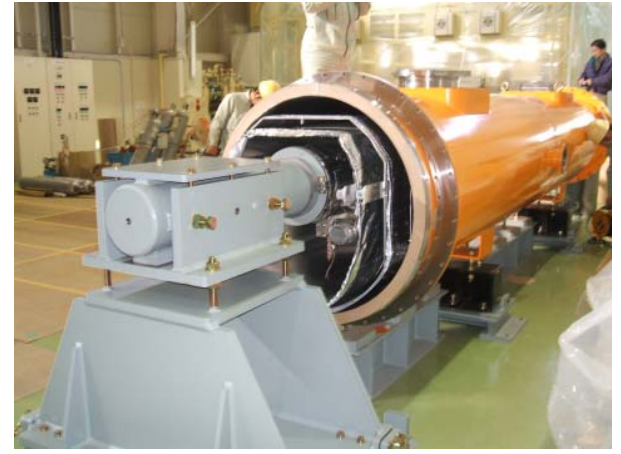

Figure 7: Insertion into the vacuum vessels

\section{Movement to the Underground Tunnel and Final Assembly}

After each of the two cryostats was completed, both were carried into the underground tunnel beneath the STF Linac Building, leveled to $0.1 \mathrm{~mm}$ or less in the vertical direction, and then underwent final assembly. The main tasks were to connect the piping at the end and middle of the cryostats, treat the measuring lines, and install the shield plates. In parallel with these tasks, KEK installed the beam pipes, WPM pipes and wires, room-temperature couplers, and other components.

Finally, the large-diameter bellows was installed between both cryostats to connect them, thus completing the STF cryomodule.

The last positional measurement was conducted at KEK. The STF cryomodule is now being considered and evaluated in terms of assembly precision along with records of the manufacturing process.

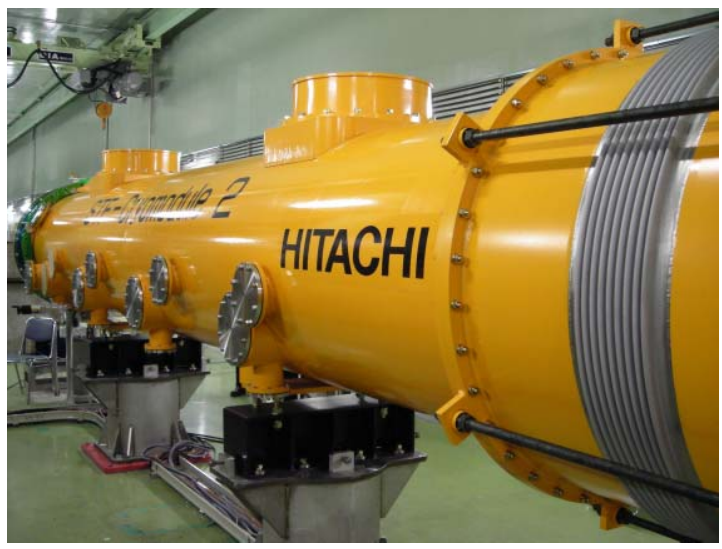

Figure 8: Completed STF cryomodule

As of May 2007, connections with the super-fluid helium cryogenic system are now being made in preparation for cryogenic testing. After that, the evaluation test as a cryostat will be carried out.

\section{SUMMARY}

The STF cryomodule was manufactured and underwent final assembly for the purpose of R\&D oriented toward ILC. This project made it possible to obtain valuable knowledge and experience regarding the processes of manufacturing and assembly, and use this knowledge and experience as the first step toward future design and manufacturing.

\section{ACKNOWLEDGMENTS}

We wish to express our thanks to the many personnel concerned at DESY, INFN, and KEK for their useful advice and cooperation.

\section{REFERENCES}

[1] K. Saito "SC Test Facilities toward the ILC", EPAC'06, Edinburgh, June 2006.

[2] K. Tsuchiya, et al. "Cryomodule Development for Superconducting RF Test Facility (STF) at KEK", EPAC'06, Edinburgh, June 2006. 